\title{
Interaction between Hydromagnetic Waves and the Anisotropically Conducting Ionosphere
}

\author{
M. ITONAGA ${ }^{1}$, A. YoshiKaWA ${ }^{2}$, and T.-I. KitAMURA ${ }^{3}$ \\ ${ }^{1}$ Computation Center, Kyushu University, Fukuoka 812, Japan \\ ${ }^{2}$ Department of Physics, Kyushu University, Fukuoka. 812, Japan \\ ${ }^{3}$ Department of Earth and Planetary Sciences, Kyushu University, Fukuoka 812, Japan
}

(Received October 23, 1994; Revised April 24, 1995; Accepted May 1, 1995)

\begin{abstract}
The self-consistent ionospheric boundary conditions, which describe the interaction between hydromagnetic (HM) waves and the anisotropically conducting ionosphere, have been derived in the form convenient for the eigenmode analysis of coupled HM waves in the magnetosphere-ionosphere system. These conditions consist of (a) the continuity of the horizontal perturbation electric field across the ionosphere, (b) the divergence of a vector formula relating a jump of the horizontal perturbation magnetic field across the ionosphere to the sheet current flowing therein and (c) the normal component of the rotation of the vector formula to the ionosphere.

In order to emphasize a significant role of the derived self-consistent conditions, under the assumption that there exists no parallel inhomogeneity but a radial one of the Alfvén velocity $V_{\mathrm{A}}$ and the ionospheric conductivities in both hemispheres are uniform and symmetric, the coupling between the axisymmetric fast and Alfvén waves via the ionospheric Hall current has been investigated in case of no energy dissipation (or zero Pedersen conductivity). It is suggested that for a given fundamental eigenfrequency, the Alfvén mode perturbation is sharply enhanced with a finite amplitude at a field line position which is deviated from the position predicted by the classical field line resonance theory that the resonant frequency is determined only from the distribution of $V_{\mathrm{A}}$ along the field line and its length $\ell_{\|}$between the northern and southern ionospheres. Such a deviation is due to the divergent Hall current and occurs toward the direction in which $V_{\mathrm{A}}$ increases. Further, the Alfvén mode perturbation has no phase shift across the enhancement position. It is also suggested that the parallel wavelength of the fast mode perturbation is slightly greater than $2 \ell_{\|}$in the region with smaller $V_{\mathrm{A}}$ and $2 \ell_{\|} / 3$ in the region with larger $V_{\mathrm{A}}$, respectively, and it changes rapidly from $\sim 2 \ell_{\|}$to $\sim 2 \ell_{\|} / 3$ through $\ell_{\|}$as $V_{\mathrm{A}}$ grows large, where the wavelength $\ell_{\|}$appears just at the enhancement position.
\end{abstract}

\section{Introduction}

The interaction between hydromagnetic (HM) waves and the ionosphere has been studied by many researchers (e.g., Newton et al., 1978; Allan and Knox, 1979; Glassmeier, 1983, 1984; Kivelson and Southwood, 1988). Allan and Knox (1979) found by using the boundary conditions describing a jump of horizontal magnetic fields across the ionosphere that the axisymmetric toroidal and poloidal modes in a dipole magnetic field interact via the ionospheric Hall current. They also discovered a new set of harmonics of a quarter-wave fundamental with longer eigenperiods than the classical half-wave one for a given magnetic shell in the case of zero Hall conductivity. On the other hand, Glassmeier $(1983,1984)$ used ionospheric boundary conditions

$$
\begin{gathered}
\Sigma_{\mathrm{P}} \nabla \cdot \mathbf{E}_{\perp}^{\mathrm{I}}+\nabla_{\perp} \Sigma_{\mathrm{P}} \cdot \mathbf{E}_{\perp}^{\mathrm{I}}+\left(\nabla_{\perp} \Sigma_{\mathrm{H}} \times \mathbf{E}_{\perp}^{\mathrm{I}}\right)_{z}=-\frac{1}{\mu_{0}} \lim _{z \downarrow 0}\left(\nabla \times \mathbf{b}^{\mathrm{M}}\right)_{z}, \\
\left(\nabla \times \mathbf{E}_{\perp}^{\mathrm{I}}\right)_{z}=0
\end{gathered}
$$


to investigate the influence of non-uniform ionospheric conductivity distributions on an Alfvén wave incident from the magnetosphere (Eq. (1) is slightly different from the original form in his papers). Here, $\mathbf{E}_{\perp}^{\mathrm{I}}$ is the horizontal electric field in the ionosphere and $\mathbf{b}^{\mathrm{M}}$ the magnetic field in the magnetosphere, and the $z$ axis is vertically upward, the subscript $z$ denotes the $z$ component of a vector quantity and $z=0$ corresponds to the thin sheet ionosphere (see Fig. 1), and $\Sigma_{\mathrm{P}}$ and $\Sigma_{\mathrm{H}}$ are the height-integrated Pedersen and Hall conductivities, respectively. When the ionosphere is uniform, Eqs. (1) and (2) are equivalent to a single vector condition (Kivelson and Southwood, 1988)

$$
\lim _{z \downarrow 0} \mathbf{b}^{\mathrm{M}}=-\mu_{0} \Sigma_{\mathrm{P}} \mathbf{e}_{z} \times \mathbf{E}_{\perp}^{\mathrm{I}},
$$

where $\mathbf{e}_{z}$ is the unit vector parallel to the $z$ axis. Using this vector condition, Newton et al. (1978) examined the damping of standing Alfvén waves due to the Joule dissipation.

Some observational data suggest that field line resonances are often dominated by a disctrete spectral structure (e.g., Samson and Rostoker, 1972; Kivelson et al., 1984), and this fact gave stimulus to the exploration of the global cavity mode theory (Kivelson and Southwood, 1985, 1986). According to this theory, impulsive sources excite poloidal-mode cavity resonances which then couple energy into toroidal-mode field line resonances. Only those field lines where the resonant frequencies match the eigenfrequencies of the global cavity modes are excited. In the classical field line resonance theory (e.g., Orr and Matthew, 1971), the resonant frequency has been considered to be determined only from the distribution of Alfvén velocity along the field line and its length between the northern and southern ionospheres. The coupled HM waves in the magnetosphere have been simulated numerically under the assumption of the perfectly conducting ionosphere (e.g., Lee and Lysak, 1989, 1990; Fujita and Patel, 1992) or in consideration of the dissipation effect of the ionosphere corresponding to Eq. (3) (e.g., Allan et al., 1985, 1986). In order to examine the transmission of HM waves through the ionosphere, however, it is of essential importance to take into account the anisotropy of the ionospheric conductivity. Using a simple model for the magnetosphere bounded by the anisotropically conducting ionosphere and the insulating atmosphere, Fujita (1993) has performed an eigenmode analysis of the coupled HM oscillations and has reconsidered the coupling between the axisymmetric fast (compressional) and Alfvén waves via the ionospheric Hall current which was first found by Allan and Knox (1979). Then, it has been shown that the Alfvén mode perturbation is enhanced along the resonant field line but the enhancement in the fast mode one does not occur. Yoshikawa et al. $(1994,1995)$ have also developed a computer simulation code for the eigenmode analysis of coupled HM waves in the magnetosphere-ionosphere system with the ionospheric boundary conditions including the Hall

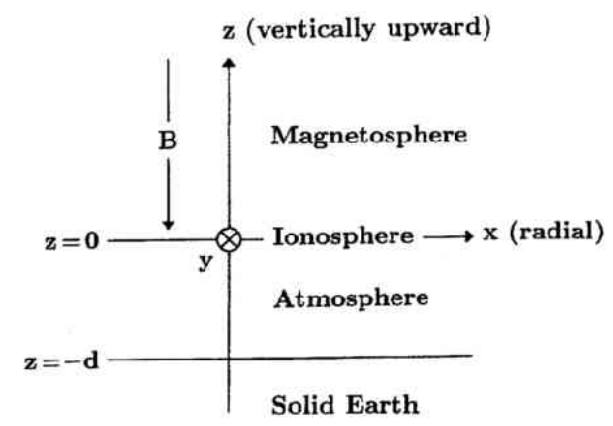

Fig. 1. Model space in the northern hemisphere divided into four regions. The ambient magnetic field $\mathbf{B}$ is assumed to be directed vertically downward. 
effect. Fujita (1993) treated the solution in the insulating atmosphere directly, while Yoshikawa et al. $(1994,1995)$ incorporated it into the ionospheric boundary conditions. They have found that the eigenperiod of field line oscillation is effectively controlled by the ionospheric Hall conductivity in the resonance region.

In the present paper we will derive the self-consistent ionospheric boundary conditions which describe the interaction between HM waves and the anisotropically conducting ionosphere and have been used in the simulation code of Yoshikawa et al. $(1994,1995)$. Then, to emphasize a significant role of the derived self-consistent conditions, we will examine the coupling between the axisymmetric fast and Alfvén waves via the ionospheric Hall current.

\section{Self-Consistent Ionospheric Boundary Conditions}

In the northern hemisphere the space is divided into four horizontally stratified reigions: the magnetosphere, the ionosphere, the atmosphere, and the solid earth (Fig. 1). The magnetosphere $(z>0)$ is regarded as an HM region filled up with the cold magnetized plasma, the ionosphere $(z=0)$ as an anisotropically conducting thin sheet parallel to the $x-y$ plane, the atmosphere $(-d<z<0)$ as a vacuum region, and the solid earth $(z<-d)$ as a perfect conductor with its surface also parallel to the $x-y$ plane. The $x$ direction corresponds to the radial one at the equatorial plane of the magnetosphere. Since we are concerned with HM waves in high latitudes, the ambient magnetic field $\mathbf{B}$ is assumed to be directed vertically downward.

Across the ionosphere $(z=0)$ the horizontal perturbation electric field $\mathbf{E}_{\perp}$ is continuous, but a jump of the horizontal perturbation magnetic field $\mathbf{b}_{\perp}$ takes place owing to the sheet current $\mathbf{J}_{\perp}^{\mathbf{I}}$ flowing therein. Thus, the ionospheric boundary conditions are:

$$
\begin{gathered}
\lim _{z \downarrow 0} \mathbf{E}_{\perp}^{\mathrm{M}}=\mathbf{E}_{\perp}^{\mathrm{I}}=\lim _{z \uparrow 0} \mathbf{E}_{\perp}^{\mathrm{A}}, \\
\mu_{0} \mathbf{J}_{\perp}^{\mathrm{I}}=\mathbf{e}_{z} \times\left(\lim _{z \downarrow 0} \mathbf{b}_{\perp}^{\mathrm{M}}-\lim _{z \uparrow 0} \mathbf{b}_{\perp}^{\mathrm{A}}\right),
\end{gathered}
$$

where the superscripts $\mathrm{M}, \mathrm{I}$ and A denote the quantity in the magnetosphere, ionosphere and atmosphere, respectively, and $\mathbf{J}_{\perp}^{\mathrm{I}}$ is given in terms of $\mathbf{E}_{\perp}^{\mathrm{I}}$ as

$$
\mathrm{J}_{\perp}^{\mathrm{I}}=\Sigma_{\mathrm{P}} \mathbf{E}_{\perp}^{\mathrm{I}}+\Sigma_{\mathrm{H}} \mathbf{E}_{\perp}^{\mathrm{I}} \times \mathbf{e}_{z} .
$$

We take

$$
\nabla \cdot \mathbf{J}_{\perp}^{\mathrm{I}}=-\lim _{z \downarrow 0} j_{z}^{\mathrm{M}}
$$

from the divergence of Eq. (5) and

$$
\left(\nabla \times \mathbf{J}_{\perp}^{\mathrm{I}}\right)_{z}=-\frac{1}{\mu_{0}}\left(\lim _{z \downarrow 0} \frac{\partial b_{z}^{\mathrm{M}}}{\partial z}-\lim _{z \uparrow 0} \frac{\partial b_{z}^{\mathrm{A}}}{\partial z}\right)
$$

from the rotation of Eq. (5). Here,

$$
j_{z}^{\mathrm{M}}=\frac{1}{\mu_{0}}\left(\nabla \times \mathbf{b}_{\perp}^{\mathrm{M}}\right)_{z}
$$

is the vertical current density in the magnetosphere, and it should be noted that the vertical current vanishes in the atmosphere. Now, we assume a time variation of the form $\mathrm{e}^{p t}$ with a complex constant $p$. Then, we have

$$
j_{z}^{\mathrm{M}}=-\frac{1}{\mu_{0} p} \frac{\partial}{\partial z}\left(\nabla \cdot \mathbf{E}_{\perp}^{\mathrm{M}}\right)
$$


from

$$
\mu_{0} \frac{\partial j_{z}^{\mathrm{M}}}{\partial t}=-\left[\nabla \times\left(\nabla \times \mathbf{E}_{\perp}^{\mathrm{M}}\right)\right]_{z}=-\frac{\partial}{\partial z}\left(\nabla \cdot \mathbf{E}_{\perp}^{\mathrm{M}}\right)
$$

and

$$
b_{z}^{\mathrm{M}, \mathrm{A}}=-\frac{1}{p}\left(\nabla \times \mathrm{E}_{\perp}^{\mathrm{M}, \mathrm{A}}\right)_{z}
$$

from

$$
\nabla \times \mathbf{E}=-\frac{\partial \mathbf{b}}{\partial t}
$$

Substituting Eqs. (6) and (10) into Eq. (7) and Eqs. (6) and (12) into Eq. (8), we eventually attain to two scalar boundary conditions

$$
\begin{aligned}
& \Sigma_{\mathrm{P}} \nabla \cdot \mathbf{E}_{\perp}^{\mathrm{I}}+\Sigma_{\mathrm{H}}\left(\nabla \times \mathbf{E}_{\perp}^{\mathrm{I}}\right)_{z}+\nabla_{\perp} \Sigma_{\mathrm{P}} \cdot \mathbf{E}_{\perp}^{\mathrm{I}}+\left(\nabla_{\perp} \Sigma_{\mathrm{H}} \times \mathbf{E}_{\perp}^{\mathrm{I}}\right)_{z} \\
& \quad=\frac{1}{\mu_{0} p} \lim _{z \downarrow 0} \frac{\partial}{\partial z}\left(\nabla \cdot \mathbf{E}_{\perp}^{\mathrm{M}}\right), \\
& \Sigma_{\mathrm{P}}\left(\nabla \times \mathbf{E}_{\perp}^{\mathrm{I}}\right)_{z}-\Sigma_{\mathrm{H}} \nabla \cdot \mathbf{E}_{\perp}^{\mathrm{I}}+\left(\nabla_{\perp} \Sigma_{\mathrm{P}} \times \mathbf{E}_{\perp}^{\mathrm{I}}\right)_{z}-\nabla_{\perp} \Sigma_{\mathrm{H}} \cdot \mathbf{E}_{\perp}^{\mathrm{I}} \\
& \quad=\frac{1}{\mu_{0} p}\left[\lim _{z \downarrow 0} \frac{\partial}{\partial z}\left(\nabla \times \mathbf{E}_{\perp}^{\mathrm{M}}\right)_{z}-\lim _{z \uparrow 0} \frac{\partial}{\partial z}\left(\nabla \times \mathbf{E}_{\perp}^{\mathrm{A}}\right)_{z}\right],
\end{aligned}
$$

which are equivalent to Eq. (5). On the assumption that Eq. (2) holds, Eq. (14) leads to Eq. (1).

If it is possible to express the second term in the righthand side of Eq. (15) in terms of $\left(\nabla \times \mathbf{E}_{\perp}^{\mathrm{I}}\right)_{z}$, the expression will be convenient for the eigenmode analysis of coupled HM waves in the magnetosphere-ionosphere system because the wave equation in the atmosphere, which is quite different from that in the magnetosphere, can be removed from the analysis system. The governing equation for $\left(\nabla \times \mathbf{E}_{\perp}^{\mathrm{A}}\right)_{z}$ is

$$
\left(\nabla^{2}-\frac{p^{2}}{c^{2}}\right)\left(\nabla \times \mathbf{E}_{\perp}^{\mathrm{A}}\right)_{z}=0
$$

where $c$ is the light velocity. Assuming a horizontal spatial variation in the form

$$
\left(\nabla \times \mathbf{E}_{\perp}^{\mathrm{A}}\right)_{z}(x, y, z)=\sum_{m=-M}^{M} \sum_{n=-N}^{N}\left(\nabla \times \mathbf{E}_{\perp}^{\mathrm{A}}\right)_{z}^{(m, n)}(z) \mathrm{e}^{i\left(m k_{x} x+n k_{y} y\right)},
$$

where $k_{x}$ and $k_{y}$ are positive constants, we have

$$
\left[\frac{d^{2}}{d z^{2}}-\left(m^{2}{k_{x}}^{2}+n^{2} k_{y}{ }^{2}+\frac{p^{2}}{c^{2}}\right)\right]\left(\nabla \times \mathbf{E}_{\perp}^{\mathrm{A}}\right)_{z}^{(m, n)}(z)=0
$$

from Eq. (16). Under the condition that $\left(\nabla \times \mathbf{E}_{\perp}^{\mathrm{A}}\right)_{z}$ vanishes on the surface $(z=-d)$ of the perfectly conducting earth, the solution of Eq. (18) becomes

$$
\left(\nabla \times \mathbf{E}_{\perp}^{\mathrm{A}}\right)_{z}^{(m, n)}(z)=\left(\nabla \times \mathbf{E}_{\perp}^{\mathrm{A}}\right)_{z}^{(m, n)}(0) \frac{\sinh \left[\gamma_{m, n}(z+d)\right]}{\sinh \left(\gamma_{m, n} d\right)},
$$

where

$$
\gamma_{m, n}=\sqrt{m^{2} k_{x}^{2}+n^{2} k_{y}^{2}+\frac{p^{2}}{c^{2}}}
$$


Then, we take

$$
\begin{aligned}
\frac{\partial}{\partial z} & \left(\nabla \times \mathbf{E}_{\perp}^{\mathrm{A}}\right)_{z}(x, y, z) \\
& =\sum_{m=-M}^{M} \sum_{n=-N}^{N}\left\{\gamma_{m, n}\left(\nabla \times \mathbf{E}_{\perp}^{\mathrm{A}}\right)_{z}^{(m, n)}(0) \frac{\cosh \left[\gamma_{m, n}(z+d)\right]}{\sinh \left(\gamma_{m, n} d\right)}\right\} \mathrm{e}^{i\left(m k_{x} x+n k_{y} y\right)}
\end{aligned}
$$

from Eqs. (17) and (19). On the assumption that

$$
\sqrt{k_{x}^{2}+k_{y}^{2}} \gg\left|\frac{p}{c}\right|, \quad d \sqrt{M^{2} k_{x}^{2}+N^{2} k_{y}^{2}} \ll 1,
$$

the approximation

$$
\frac{\cosh \left[\gamma_{m, n}(z+d)\right]}{\sinh \left(\gamma_{m, n} d\right)} \simeq \frac{1}{\gamma_{m, n} d}
$$

holds for all $|m| \leq M$ and $|n| \leq N$. Then, we obtain

$$
\lim _{z \uparrow 0} \frac{\partial}{\partial z}\left(\nabla \times \mathbf{E}_{\perp}^{\mathrm{A}}\right)_{z} \simeq \frac{1}{d}\left(\nabla \times \mathbf{E}_{\perp}^{\mathrm{I}}\right)_{z}
$$

from Eqs. (17), (20) and (21). Equation (22) will be valid for low-frequency HM waves with a scale of the horizontal spatial variation which is sufficiently large compared with $d$. Thus, we obtain a boundary condition

$$
\begin{aligned}
\Sigma_{\mathrm{P}} & \left(\nabla \times \mathbf{E}_{\perp}^{\mathrm{I}}\right)_{z}-\Sigma_{\mathrm{H}} \nabla \cdot \mathbf{E}_{\perp}^{\mathrm{I}}+\left(\nabla_{\perp} \Sigma_{\mathrm{P}} \times \mathbf{E}_{\perp}^{\mathrm{I}}\right)_{z}-\nabla_{\perp} \Sigma_{\mathbf{H}} \cdot \mathbf{E}_{\perp}^{\mathrm{I}} \\
& =\frac{1}{\mu_{0} p}\left[\lim _{z \downarrow 0} \frac{\partial}{\partial z}\left(\nabla \times \mathbf{E}_{\perp}^{\mathrm{M}}\right)_{z}-\frac{1}{d}\left(\nabla \times \mathbf{E}_{\perp}^{\mathrm{I}}\right)_{z}\right]
\end{aligned}
$$

which is preferable in the eigenmode analysis of coupled HM waves in the magnetosphereionosphere system to Eq. (15).

The physical meanings of the ionospheric boundary conditions (Eqs. (14) and (23)) are interpreted as follows. When an Alfvén wave with the divergent electric field $\left(\nabla \cdot \mathbf{E}_{\perp}^{\mathrm{M}} \neq 0\right)$ is incident from the magnetosphere, the magnetic fields of the incident and reflected Alfven waves are shielded from the ground by the ionospheric divergent current and the waves with the rotational electric field $\left(\left(\nabla \times \mathbf{E}_{\perp}^{\mathrm{M}, \mathrm{A}}\right)_{z} \neq 0\right)$ are simultaneously transmitted above and below the ionosphere by the ionospheric rotational current. On the other hand, when a fast (compressional) wave with the rotational electric field is incident from the magnetosphere, its magnetic field is transmitted to the ground through the ionosphere with a modification due to the ionospheric rotational current and the magnetic field of the reflected Alfvén wave simultaneously generated by the ionospheric divergent current is also shielded from the ground.

\section{Mode Coupling via the Ionospheric Hall Current}

In order to emphasize a significant role of the derived self-consistent ionospheric boundary conditions, assuming that there exists no parallel inhomogeneity but a radial one of the Alfvén velocity $V_{\mathrm{A}}\left(\partial V_{\mathrm{A}} / \partial x \neq 0\right)$, we examine the coupling between the axisymmetric fast and Alfvén waves via the ionospheric Hall current. In the axisymmetric case $(\partial / \partial y \equiv 0)$ the equations that the electric field $\mathbf{E}_{\perp}^{\mathrm{M}}$ should satisfy become

$$
\left(\frac{\partial^{2}}{\partial z^{2}}-\frac{p^{2}}{V_{\mathrm{A}}^{2}}\right) E_{x}^{\mathrm{M}}=0
$$




$$
\left(\frac{\partial^{2}}{\partial x^{2}}+\frac{\partial^{2}}{\partial z^{2}}-\frac{p^{2}}{V_{\mathrm{A}}^{2}}\right) E_{y}^{\mathrm{M}}=0 .
$$

Solving Eq. (24), we have

$$
E_{x}^{\mathrm{M}}(x, z)=F^{(1)}(x) \mathrm{e}^{\left(p / V_{\mathrm{A}}\right)\left(z-\ell_{\|} / 2\right)}+F^{(2)}(x) \mathrm{e}^{-\left(p / V_{\mathrm{A}}\right)\left(z-\ell_{\|} / 2\right)}
$$

as a solution, where $\ell_{\|}$is the length of field line between the northern and southern ionospheres and it is assmued to be constant throughout the magnetosphere. It should be noted that $F^{(1)}$ and $F^{(2)}$ are determined from the ionospheric boundary conditions. On the other hand, $E_{y}^{\mathrm{M}}$ can be written in the form

$$
E_{y}^{\mathrm{M}}(x, z)=\sum_{n=0}^{\infty} G_{n}(x)\left(z-\frac{\ell_{\|}}{2}\right)^{n},
$$

where $G_{n}$ 's are determined from

$$
\left(\frac{d^{2}}{d x^{2}}-\frac{p^{2}}{V_{\mathrm{A}}^{2}}\right) G_{n}+(n+1)(n+2) G_{n+2}=0 \quad(n=0,1, \ldots)
$$

with appropriate inner and outer boundary conditions in the radial direction (e.g., $G_{n}=0$ at both boundaries for all $n$ ). Since it is assumed that the ambient magnetic field penetrates the ionosphere vertically, the inner and outer boundaries, for instance, correspond to the plasmapause and the magnetopause, respectively.

Under the assumption that the ionospheric conductivities in both hemispheres are uniform and symmetric, applying the boundary conditions (Eqs. (14) and (23)) in the northern ionosphere and the corresponding ones in the southern ionosphere to $\mathbf{E}_{\perp}^{\mathrm{M}}$ given by Eqs. (26) and (27), we obtain

$$
\begin{aligned}
\frac{d}{d x} & {\left[\Sigma_{\mathrm{P}}\left(F^{(1)} \mathrm{e}^{-p \ell_{\|} / 2 V_{\mathrm{A}}}+F^{(2)} \mathrm{e}^{p \ell_{\|} / 2 V_{\mathrm{A}}}\right)+\Sigma_{\mathrm{H}} \sum_{n=0}^{\infty}\left(-\frac{\ell_{\|}}{2}\right)^{n} G_{n}\right.} \\
& \left.-\Sigma_{\mathrm{A}}\left(F^{(1)} \mathrm{e}^{-p \ell_{\|} / 2 V_{\mathrm{A}}}-F^{(2)} \mathrm{e}^{p \ell_{\|} / 2 V_{\mathrm{A}}}\right)\right]=0, \\
\frac{d}{d x} & {\left[\Sigma_{\mathrm{P}} \sum_{n=0}^{\infty}\left(-\frac{\ell_{\|}}{2}\right)^{n} G_{n}-\Sigma_{\mathrm{H}}\left(F^{(1)} \mathrm{e}^{-p \ell_{\|} / 2 V_{\mathrm{A}}}+F^{(2)} \mathrm{e}^{p \ell_{\|} / 2 V_{\mathrm{A}}}\right)\right.} \\
& \left.-\frac{1}{\mu_{0} p} \sum_{n=1}^{\infty} n\left(-\frac{\ell_{\|}}{2}\right)^{n-1} G_{n}+\frac{1}{\mu_{0} p d} \sum_{n=0}^{\infty}\left(-\frac{\ell_{\|}}{2}\right)^{n} G_{n}\right]=0
\end{aligned}
$$

from the northern boundary conditions and

$$
\begin{aligned}
& \frac{d}{d x}[ \Sigma_{\mathrm{P}}\left(F^{(1)} \mathrm{e}^{p \ell_{\|} / 2 V_{\mathrm{A}}}+F^{(2)} \mathrm{e}^{-p \ell_{\|} / 2 V_{\mathrm{A}}}\right)+\Sigma_{\mathrm{H}} \sum_{n=0}^{\infty}\left(\frac{\ell_{\|}}{2}\right)^{n} G_{n} \\
&\left.+\Sigma_{\mathrm{A}}\left(F^{(1)} \mathrm{e}^{p \ell_{\|} / 2 V_{\mathrm{A}}}-F^{(2)} \mathrm{e}^{-p \ell_{\mathbb{I}} / 2 V_{\mathrm{A}}}\right)\right]=0, \\
& \frac{d}{d x}\left[\Sigma_{\mathrm{P}} \sum_{n=0}^{\infty}\left(\frac{\ell_{\|}}{2}\right)^{n} G_{n}-\Sigma_{\mathrm{H}}\left(F^{(1)} \mathrm{e}^{p \ell_{\|} / 2 V_{\mathrm{A}}}+F^{(2)} \mathrm{e}^{-p \ell_{\|} / 2 V_{\mathrm{A}}}\right)\right. \\
&\left.+\frac{1}{\mu_{0} p} \sum_{n=1}^{\infty} n\left(\frac{\ell_{\|}}{2}\right)^{n-1} G_{n}+\frac{1}{\mu_{0} p d} \sum_{n=0}^{\infty}\left(\frac{\ell_{\|}}{2}\right)^{n} G_{n}\right]=0
\end{aligned}
$$


from the southern boundary conditions, where $\Sigma_{\mathrm{A}}=1 / \mu_{0} V_{\mathrm{A}}$ is the Alfvén wave conductance. It is seen from comparing Eqs. (29) and (30) with Eqs. $\left(29^{\prime}\right)$ and $\left(30^{\prime}\right)$ that there can exist only two cases:

$$
F^{(1)}=F^{(2)}=F / 2, \quad G_{2 n+1}=0 \quad(n=0,1, \ldots) \quad(\text { symmetric case })
$$

or

$$
F^{(1)}=-F^{(2)}=F / 2, \quad G_{2 n}=0 \quad(n=0,1, \ldots) \quad(\text { antisymmetric case) }
$$

when $\Sigma_{\mathrm{H}} \neq 0$. The oscillating electric field has loops and nodes at the equatorial plane $\left(z=\ell_{\|} / 2\right)$ in the symmetric and antisymmetric cases, respectively.

In the present study we consider only the symmetric case. In this case Eqs. (29) and (30) (or Eqs. $\left(29^{\prime}\right)$ and $\left(30^{\prime}\right)$ ) become

$$
\begin{gathered}
\frac{d}{d x}\left\{\left[\Sigma_{\mathrm{P}} \cosh \left(\frac{p \ell_{\|}}{2 V_{\mathrm{A}}}\right)+\Sigma_{\mathrm{A}} \sinh \left(\frac{p \ell_{\|}}{2 V_{\mathrm{A}}}\right)\right] E_{x}^{\mathrm{M}}\left(x, \frac{\ell_{\|}}{2}\right)+\Sigma_{\mathrm{H}} E_{y}^{\mathrm{M}}(x, 0)\right\}=0, \\
\frac{d}{d x}\left[-\Sigma_{\mathrm{H}} \cosh \left(\frac{p \ell_{\|}}{2 V_{\mathrm{A}}}\right) E_{x}^{\mathrm{M}}\left(x, \frac{\ell_{\|}}{2}\right)+\left(\Sigma_{\mathrm{P}}+\frac{1}{\mu_{0} p d}\right) E_{y}^{\mathrm{M}}(x, 0)-\frac{1}{\mu_{0} p}\left(\frac{\partial E_{y}^{\mathrm{M}}}{\partial z}\right)(x, 0)\right]=0,
\end{gathered}
$$

respectively, where

$$
\begin{aligned}
& E_{x}^{\mathrm{M}}\left(x, \frac{\ell_{\|}}{2}\right)=F(x), \\
& E_{y}^{\mathrm{M}}(x, 0) \\
& \left(\frac{\partial E_{y}^{\mathrm{M}}}{\partial z}\right)(x, 0)=-\sum_{n=0}^{\infty}\left(\frac{\ell_{\|}}{2}\right)^{2 n} G_{2 n}(x),
\end{aligned}
$$

While we take

$$
\begin{aligned}
& {\left[\Sigma_{\mathrm{P}} \cosh \left(\frac{p \ell_{\|}}{2 V_{\mathrm{A}}}\right)+\Sigma_{\mathrm{A}} \sinh \left(\frac{p \ell_{\|}}{2 V_{\mathrm{A}}}\right)\right] E_{x}^{\mathrm{M}}\left(x, \frac{\ell_{\|}}{2}\right)+\Sigma_{\mathrm{H}} E_{y}^{\mathrm{M}}(x, 0)} \\
& =\text { const. } \equiv C_{1} \\
& -\Sigma_{\mathrm{H}} \cosh \left(\frac{p \ell_{\|}}{2 V_{\mathrm{A}}}\right) E_{x}^{\mathrm{M}}\left(x, \frac{\ell_{\|}}{2}\right)+\left(\Sigma_{\mathrm{P}}+\frac{1}{\mu_{0} p d}\right) E_{y}^{\mathrm{M}}(x, 0)-\frac{1}{\mu_{0} p}\left(\frac{\partial E_{y}^{\mathrm{M}}}{\partial z}\right)(x, 0) \\
& =\text { const. } \equiv C_{2}
\end{aligned}
$$

from Eqs. (31) and (32), it is necessary for Eqs. (33) and (34) to hold simultaneously that the fast mode perturbation $E_{y}^{\mathrm{M}}$ satisfies the following equation

$$
\begin{gathered}
{\left[\Sigma_{\mathrm{P}} \cosh \left(\frac{p \ell_{\|}}{2 V_{\mathrm{A}}}\right)+\Sigma_{\mathrm{A}} \sinh \left(\frac{p \ell_{\|}}{2 V_{\mathrm{A}}}\right)\right]\left[\left(\Sigma_{\mathrm{P}}+\frac{1}{\mu_{0} p d}\right) E_{y}^{\mathrm{M}}(x, 0)-\frac{1}{\mu_{0} p}\left(\frac{\partial E_{y}^{\mathrm{M}}}{\partial z}\right)(x, 0)\right]} \\
+\Sigma_{\mathrm{H}}^{2} \cosh \left(\frac{p \ell_{\|}}{2 V_{\mathrm{A}}}\right) E_{y}^{\mathrm{M}}(x, 0) \\
=C_{1} \Sigma_{\mathrm{H}} \cosh \left(\frac{p \ell_{\|}}{2 V_{\mathrm{A}}}\right)+C_{2}\left[\Sigma_{\mathrm{P}} \cosh \left(\frac{p \ell_{\|}}{2 V_{\mathrm{A}}}\right)+\Sigma_{\mathrm{A}} \sinh \left(\frac{p \ell_{\|}}{2 V_{\mathrm{A}}}\right)\right]
\end{gathered}
$$

in the ionosphere. Further, if we assume that $G_{2 n}=0$ at the inner and outer boundaries for all $n$, that is, $E_{y}^{\mathrm{M}}=0$ and $\partial E_{y}^{\mathrm{M}} / \partial z=0$ at both boundaries, it is also necessary that $C_{1}=C_{2}=0$ so 
far as $V_{\mathrm{A}}$ has different values at both boundaries. Thus, $G_{2 n}$ 's are constrained by the condition

$$
\begin{gathered}
{\left[\Sigma_{\mathrm{P}} \cosh \left(\frac{p \ell_{\|}}{2 V_{\mathrm{A}}}\right)+\Sigma_{\mathrm{A}} \sinh \left(\frac{p \ell_{\|}}{2 V_{\mathrm{A}}}\right)\right]\left[\left(\Sigma_{\mathrm{P}}+\frac{1}{\mu_{0} p d}\right) E_{y}^{\mathrm{M}}(x, 0)-\frac{1}{\mu_{0} p}\left(\frac{\partial E_{y}^{\mathrm{M}}}{\partial z}\right)(x, 0)\right]} \\
+\Sigma_{\mathrm{H}}{ }^{2} \cosh \left(\frac{p \ell_{\|}}{2 V_{\mathrm{A}}}\right) E_{y}^{\mathrm{M}}(x, 0)=0,
\end{gathered}
$$

which prescribes the parallel structure of the fast mode perturbation. Since it is quite difficult to obtain an eigenvalue $p$ and a set of eigenfunctions $G_{2 n}$ 's of Eq. (28) under the constraint of Eq. (36), we will develop a discussion on the subject in the following under the assumption that these ones have been given, although it is known that discretizing Eqs. (24) and (25) directly leads to a relatively simple eigenvalue problem (Fujita, 1993; Yoshikawa et al., 1994, 1995). Once $G_{2 n}$ 's have been acquired as eigenfunctions, the Alfvén mode perturbation $E_{x}^{\mathrm{M}}$ at the equatorial plane is given by Eq. (33) or Eq. (34) with $C_{1}=C_{2}=0$. It is seen from Eqs. (33) and (34) that the Alfvén mode perturbation has no singularity because the functions cosh and sinh can by no means vanish concurrently.

Let us consider the case of $\Sigma_{P}=0$ for simplification. In this case, since there is no energy dissipation, $p$ becomes purely imaginary $(p=i \omega)$. Here, letting $V_{\mathrm{A}, 0}$ denote $V_{\mathrm{A}}$ at the outer boundary of the magnetosphere, we introduce the following normalizations:

$$
\bar{x}=\frac{x}{\ell_{\|}}, \quad \bar{z}=\frac{z}{\ell_{\|}}, \quad \bar{\omega}=\frac{\omega \ell_{\|}}{V_{\mathrm{A}, 0}}, \quad \bar{\Sigma}_{\mathrm{A}}=\frac{\Sigma_{\mathrm{A}}}{\Sigma_{\mathrm{A}, 0}}, \quad \bar{\Sigma}_{\mathrm{H}}=\frac{\Sigma_{\mathrm{H}}}{\Sigma_{\mathrm{A}, 0}},
$$

where $\Sigma_{\mathrm{A}, 0}=1 / \mu_{0} V_{\mathrm{A}, 0}$. Then, Eq. (36) becomes

$$
\begin{gathered}
{\left[\frac{1}{\bar{\omega} \bar{d}} \bar{\Sigma}_{\mathrm{A}} \sin \left(\frac{\bar{\omega}}{2} \bar{\Sigma}_{\mathrm{A}}\right)+\bar{\Sigma}_{\mathrm{H}}^{2} \cos \left(\frac{\bar{\omega}}{2} \bar{\Sigma}_{\mathrm{A}}\right)\right]\left(E_{y}^{\mathrm{M}}\right)_{\text {ionosphere }}} \\
=\frac{1}{\bar{\omega}} \bar{\Sigma}_{\mathrm{A}} \sin \left(\frac{\bar{\omega}}{2} \bar{\Sigma}_{\mathrm{A}}\right)\left(\frac{\partial E_{y}^{\mathrm{M}}}{\partial \bar{z}}\right)_{\text {ionosphere }}
\end{gathered}
$$

where

$$
\left(E_{y}^{\mathrm{M}}\right)_{\text {ionosphere }}=\lim _{\bar{z} \downarrow 0} E_{y}^{\mathrm{M}}(\bar{x}, \bar{z}), \quad\left(\frac{\partial E_{y}^{\mathrm{M}}}{\partial \bar{z}}\right)_{\text {ionosphere }}=\lim _{\bar{z} \downarrow 0}\left(\frac{\partial E_{y}^{\mathrm{M}}}{\partial \bar{z}}\right)(\bar{x}, \bar{z}) .
$$

Further, we have

$$
\frac{i\left(E_{x}^{\mathrm{M}}\right)_{\text {equator }}}{\left(E_{y}^{\mathrm{M}}\right)_{\text {ionosphere }}}=-\frac{\bar{\Sigma}_{\mathrm{H}}}{\bar{\Sigma}_{\mathrm{A}} \sin \left(\frac{\bar{\omega}}{2} \bar{\Sigma}_{\mathrm{A}}\right)}
$$

from Eq. (33) with $C_{1}=0$ and also have

$$
\frac{i\left(E_{x}^{\mathrm{M}}\right)_{\text {equator }}}{\left(\partial E_{y}^{\mathrm{M}} / \partial \bar{z}\right)_{\text {ionosphere }}}=-\frac{\frac{1}{\bar{\omega}} \bar{\Sigma}_{\mathrm{H}}}{\frac{1}{\bar{\omega} \bar{d}} \bar{\Sigma}_{\mathrm{A}} \sin \left(\frac{\bar{\omega}}{2} \bar{\Sigma}_{\mathrm{A}}\right)+\bar{\Sigma}_{\mathrm{H}}{ }^{2} \cos \left(\frac{\bar{\omega}}{2} \bar{\Sigma}_{\mathrm{A}}\right)}
$$

using Eq. (37), where

$$
\left(E_{x}^{\mathrm{M}}\right)_{\text {equator }}=\lim _{\bar{z} \rightarrow 1 / 2} E_{x}^{\mathrm{M}}(\bar{x}, \bar{z}) .
$$

Note that the ratio given by Eq. (38) has real values, corresponding to a phase difference of $\pi / 2$ between $E_{x}^{\mathrm{M}}$ and $E_{y}^{\mathrm{M}}$. Here, we assume that $V_{\mathrm{A}}$ has the minimum value at the outer boundary 
and increases monotonously toward the inner boundary, meaning that $\bar{\Sigma}_{\mathrm{A}}$ is equal to 1 at the outer bounadry and decreases toward the inner boundary, and $2 \pi<\bar{\omega}<4 \pi$. This assumption means that the righthand side of Eq. (38) diverges at one field line position at most between the inner and outer boundaries. Then, although the righthand side of Eq. (38) diverges at the field line position of $\bar{\Sigma}_{\mathrm{A}}=2 \pi / \bar{\omega}$ and that of Eq. (39) at the position of $\bar{\Sigma}_{\mathrm{A}}=\bar{\Sigma}_{\mathrm{A}, \mathrm{c}}$ which satisfies

$$
\frac{1}{\bar{\omega} \bar{d}} \bar{\Sigma}_{\mathrm{A}, \mathrm{c}} \sin \left(\frac{\bar{\omega}}{2} \bar{\Sigma}_{\mathrm{A}, \mathrm{c}}\right)+\bar{\Sigma}_{\mathrm{H}}^{2} \cos \left(\frac{\bar{\omega}}{2} \bar{\Sigma}_{\mathrm{A}, \mathrm{c}}\right)=0,
$$

where the inner boundary is supposed to be located at the position of $\bar{\Sigma}_{\mathrm{A}}<2 \pi / \bar{\omega}$ and $\bar{\Sigma}_{\mathrm{A}}<\bar{\Sigma}_{\mathrm{A}, \mathrm{c}}$, these divergences never indicate that the Alfvén mode perturbation $E_{x}^{\mathrm{M}}$ has some singularities at those positions because $\left(E_{y}^{\mathrm{M}}\right)_{\text {ionosphere }}=0$ at $\bar{\Sigma}_{\mathrm{A}}=2 \pi / \bar{\omega}$ and $\left(\partial E y^{\mathrm{M}} / \partial \bar{z}\right)_{\text {ionosphere }}=0$ at $\bar{\Sigma}_{\mathrm{A}}=\bar{\Sigma}_{\mathrm{A}, c}$, as seen from Eq. (37). It should be noticed that $\left(E_{y}^{\mathrm{M}}\right)_{\text {ionosphere }}$ changes its sign across the position of $\bar{\Sigma}_{\mathrm{A}}=2 \pi / \bar{\omega}$ and $\left(\partial E y^{\mathrm{M}} / \partial \bar{z}\right)_{\text {ionosphere }}$ across the position of $\bar{\Sigma}_{\mathrm{A}}=\bar{\Sigma}_{\mathrm{A}, \mathrm{c}}$ owing to the boundedness of $\left(E_{x}^{\mathrm{M}}\right)_{\text {equator }}$ at those positions.

From Eq. (37) we have

$$
\frac{\left(\partial E_{y}^{\mathrm{M}} / \partial \bar{z}\right)_{\text {ionosphere }}}{\left(E_{y}^{\mathrm{M}}\right)_{\text {ionosphere }}}=C\left(\bar{\Sigma}_{\mathrm{A}}\right)
$$

where $C$ is given by

$$
C\left(\bar{\Sigma}_{\mathrm{A}}\right)=\bar{\omega} \frac{\bar{\Sigma}_{\mathrm{H}^{2}}}{\bar{\Sigma}_{\mathrm{A}}} \cot \left(\frac{\bar{\omega}}{2} \bar{\Sigma}_{\mathrm{A}}\right)+\frac{1}{\bar{d}} .
$$

Note that the equation $C\left(\bar{\Sigma}_{\mathrm{A}, \mathrm{c}}\right)=0$ is equivalent to Eq. (40). Figure 2 shows the dependence of the ratio $\left(\partial E_{y}^{\mathrm{M}} / \partial \bar{z}\right)_{\text {ionosphere }} /\left(E_{y}^{\mathrm{M}}\right)_{\text {ionosphere }}$ given by Eq. (41) on $\bar{\Sigma}_{\mathrm{A}}$, where the vertical line indicates the field line position of $\bar{\Sigma}_{\mathrm{A}}=2 \pi / \bar{\omega}$ and the ratio vanishes at the position of $\bar{\Sigma}_{\mathrm{A}}=\bar{\Sigma}_{\mathrm{A}, \mathrm{c}}$, corresponding to $\left(\partial E_{y}^{\mathrm{M}} / \partial \bar{z}\right)_{\text {ionosphere }}=0$ there. In this figure the parameter values of $\bar{\omega}=8.4$, $\bar{\Sigma}_{\mathrm{H}}=1$ and $\bar{d}=0.005$, which correspond to those of Fujita (1993), have been used. Then,

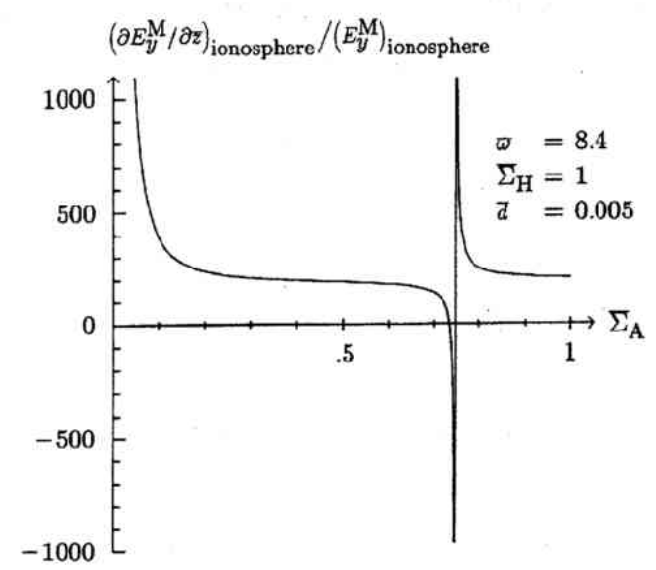

Fig. 2. Dependence of the ratio $\left(\partial E_{y}^{\mathrm{M}} / \partial \bar{z}\right)_{\text {ionosphere }} /\left(E_{y}^{\mathrm{M}}\right)_{\text {ionosphere }}$ on $\bar{\Sigma}_{\mathrm{A}}$. The vertical line indicates the field line position of $\bar{\Sigma}_{\mathrm{A}}=2 \pi / \bar{\omega}$. The ratio diverges at the positions of $\bar{\Sigma}_{\mathrm{A}}=0$ and $\bar{\Sigma}_{\mathrm{A}}=2 \pi / \bar{\omega}$, and vanishes at the position of $\bar{\Sigma}_{\mathrm{A}}=\bar{\Sigma}_{\mathrm{A}, \mathrm{c}}=0.7343964$. 
$\bar{\Sigma}_{\mathrm{A}, \mathrm{c}}=0.7343964<2 \pi / \bar{\omega}=0.7479982$. Now, let us suppose tentatively a parallel structure of the fast mode perturbation $E_{y}^{\mathrm{M}}$ given by

$$
E_{y}^{\mathrm{M}} /\left(E_{y}^{\mathrm{M}}\right)_{\text {equator }}=\cos \left[\bar{k}\left(\bar{z}-\frac{1}{2}\right)\right]
$$

with a parallel wavenumber $\bar{k}$ at each field line position. Then, since

$$
\frac{\left(\partial E_{y}^{\mathrm{M}} / \partial \bar{z}\right)_{\text {ionosphere }}}{\left(E_{y}^{\mathrm{M}}\right)_{\text {ionosphere }}}=f(\bar{k}),
$$

where

$$
f(\bar{k})=\bar{k} \tan \left(\frac{\bar{k}}{2}\right),
$$

the parallel wavenumber $\bar{k}$ is determined from

$$
f(\bar{k})=C\left(\bar{\Sigma}_{\mathrm{A}}\right) .
$$

Figure 3 displays the behavior of $f(\bar{k})$ given by Eq. (45) in the interval $-\pi<\bar{k}<3 \pi$. Here, let us assume that in the vicinity of the outer boundary, $E_{y}^{\mathrm{M}}$ has quasinodes at the ionospheric boundaries and its parallel wavelength is slightly greater than $2 \ell_{\|}$. Such an assumption will be valid for the fundamental mode. From Eq. (46), then, we obtain $\bar{k}=3.111201$ at the outer boundary $\left(\bar{\Sigma}_{\mathrm{A}}=1\right)$. As seen from Fig. 2 ,

$$
\lim _{\bar{\Sigma}_{\mathrm{A} \downarrow 0}} C\left(\bar{\Sigma}_{\mathrm{A}}\right)=+\infty, \quad \lim _{\bar{\Sigma}_{\mathrm{A}} \uparrow 2 \pi / \bar{\omega}} C\left(\bar{\Sigma}_{\mathrm{A}}\right)=-\infty, \quad \lim _{\bar{\Sigma}_{\mathrm{A} \downarrow 2 \pi / \bar{\omega}}} C\left(\bar{\Sigma}_{\mathrm{A}}\right)=+\infty .
$$

Hence, assuming that $\bar{k}$ varies continuously with $\bar{\Sigma}_{\mathrm{A}}$, we can take a branch indicating the dependence of $\bar{k}$ on $\bar{\Sigma}_{\mathrm{A}}$ for $\bar{\Sigma}_{\mathrm{A}} \leq 1$ (Fig. 4).

Figure 5 illustrates the parallel structure of the fast mode perturbation $E_{y}^{\mathrm{M}}$ given by Eq. (43) at the field line positions of $\bar{\Sigma}_{\mathrm{A}}=1,2 \pi / \bar{\omega}, 0.735, \bar{\Sigma}_{\mathrm{A}, c}, 0.734$ and 0.7 . It is obvious that the parallel structure of $E_{y}^{\mathrm{M}}$ characterized with the wavenumber $\bar{k}$ is by no means the true one. However, since $E_{y}^{\mathrm{M}}$ has loops at the equatorial plane and the ratio $\left(\partial E_{y}^{\mathrm{M}} / \partial \bar{z}\right)_{\text {ionosphere }} /\left(E_{y}^{\mathrm{M}}\right)_{\text {ionosphere }}$

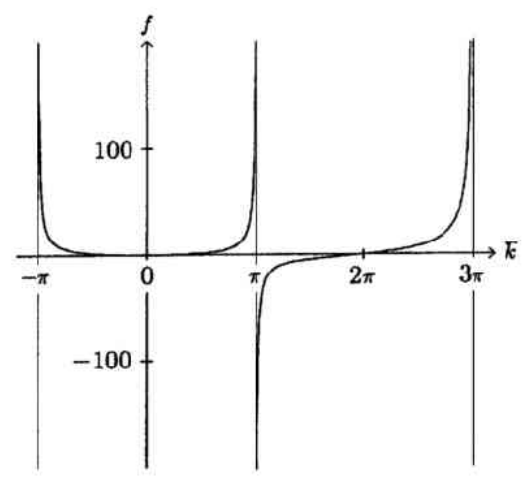

Fig. 3. Behavior of $f(\bar{k})=\bar{k} \tan (\bar{k} / 2)$ in the interval $-\pi<\bar{k}<3 \pi$. 


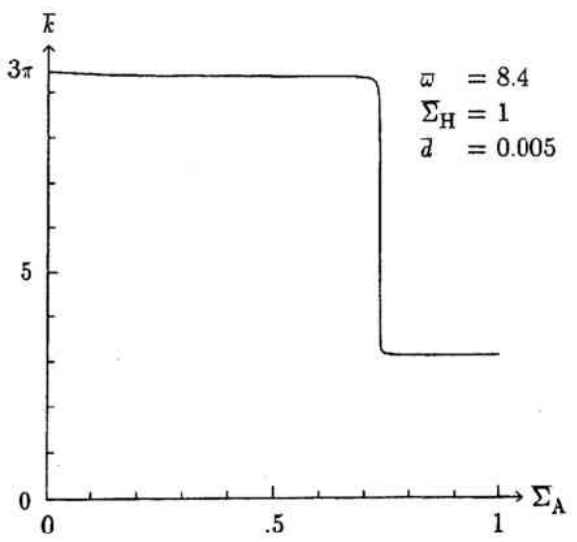

Fig. 4. Branch indicating the dependence of the parallel wavenumber $\bar{k}$ on $\bar{\Sigma}_{\mathrm{A}}$ for $\bar{\Sigma}_{\mathrm{A}} \leq 1$. Here, $\bar{k}=3.111201, \pi$ and $2 \pi$ at $\bar{\Sigma}_{\mathrm{A}}=1$ (outer boundary), $2 \pi / \bar{\omega}$ and $\bar{\Sigma}_{\mathrm{A}, \mathrm{c}}$, respectively, and $\bar{k}$ converges to $3 \pi$ as $\bar{\Sigma}_{\mathrm{A}}$ approaches 0 .

is dominated by Eq. (41), $E_{y}^{\mathrm{M}}$ will have such a parallel structure as is topologically analogous to the one given by Eq. (43). The ratio diverges as $\bar{\Sigma}_{\mathrm{A}}$ approaches $2 \pi / \bar{\omega}$, meaning that $\left(E_{y}^{\mathrm{M}}\right)_{\text {ionosphere }}=0$ at the position of $\bar{\Sigma}_{\mathrm{A}}=2 \pi / \bar{\omega}$, that is, $E_{y}^{\mathrm{M}}$ has nodes at the ionospheric boundaries and its parallel wavelength is equal to $2 \ell_{\|}$(see Fig. 5). Further, it is seen from Fig. 2 that in the region of $\bar{\Sigma}_{\mathrm{A}}<2 \pi / \bar{\omega}$, the parallel wavelength changes rapidly from $2 \ell_{\|}$to a value slightly greater than $2 \ell_{\|} / 3$ through $\ell_{\|}$as $\bar{\Sigma}_{\mathrm{A}}$ decreases across $\bar{\Sigma}_{\mathrm{A}}=\bar{\Sigma}_{\mathrm{A}, \mathrm{c}}$ (also see Fig. 5). As $\bar{\Sigma}_{\mathrm{A}}$ approaches 0 , the ratio also diverges because of $\left(E_{y}^{\mathrm{M}}\right)_{\text {ionosphere }}=0$ and the parallel wavelength converges to $2 \ell_{\|} / 3$, that is, $\bar{k}$ converges to $3 \pi$. While the ratio changes its sign across the positions of $\bar{\Sigma}_{\mathrm{A}}=2 \pi / \bar{\omega}$ and $\bar{\Sigma}_{\mathrm{A}}=\bar{\Sigma}_{\mathrm{A}, \mathrm{c}}$, these changes correspond to those in sign of $\left(E_{y}^{\mathrm{M}}\right)_{\text {ionosphere }}$ and $\left(\partial E_{y}^{\mathrm{M}} / \partial \bar{z}\right)_{\text {ionosphere }}$, respectively. Note that at the position of $\bar{\Sigma}_{\mathrm{A}}=\bar{\Sigma}_{\mathrm{A}, \mathrm{c}}, E_{y}^{\mathrm{M}}$ has loops at the ionospheric boundaries and its parallel wavelength is equal to $\ell_{\|}$(see Fig. 5).

The Alfvén mode perturbation $E_{x}^{\mathrm{M}}$ may be enhanced at the field line position of $\bar{\Sigma}_{\mathrm{A}}=2 \pi / \bar{\omega}$ or $\bar{\Sigma}_{\mathrm{A}}=\bar{\Sigma}_{\mathrm{A}, \mathrm{c}}$, as seen from Eq. (38) or Eq. (39). In the axisymmetric case we have

$$
\mu_{0} \ell_{\|} V_{\mathrm{A}, 0}\left(j_{z}^{\mathrm{M}}\right)_{\text {ionosphere }}=-\bar{\Sigma}_{\mathrm{H}} \frac{\partial}{\partial \bar{x}}\left(E_{y}^{\mathrm{M}}\right)_{\text {ionosphere }}
$$

from Eq. (7) for $\Sigma_{\mathrm{P}}=0$, where

$$
\left(j_{z}^{\mathrm{M}}\right)_{\text {ionosphere }}=\lim _{\bar{z} \downarrow 0} j_{z}^{\mathrm{M}}(\bar{x}, \bar{z}) .
$$

It may be expected that the vertical current density $\left(j_{z}^{\mathrm{M}}\right)_{\text {ionosphere }}$ vanishes at the enhancement position and its sign changes across the position. If so, it is necessary that the radial gradient of $\left(E_{y}^{\mathrm{M}}\right)_{\text {ionosphere }}$ also disappears at the position, as indicated by Eq. (47). Judging from the variation in parallel structure of the fast mode perturbation $E_{y}^{\mathrm{M}}$ as shown in Fig. 5, while the radial gradient of $\left(E_{y}^{\mathrm{M}}\right)_{\text {ionosphere }}$, accordingly, $\left(j_{z}^{\mathrm{M}}\right)_{\text {ionosphere }}$ vanishes at the position of $\bar{\Sigma}_{\mathrm{A}}=\bar{\Sigma}_{\mathrm{A}, \mathrm{c}}$ and its sign changes across this position, these do not hold for the position of $\bar{\Sigma}_{\mathrm{A}}=2 \pi / \bar{\omega}$ at all. Thus, it is suggested that $E_{x}^{\mathrm{M}}$ will be enhanced at the position of not $\bar{\Sigma}_{\mathrm{A}}=2 \pi / \bar{\omega}$ but $\bar{\Sigma}_{\mathrm{A}}=\bar{\Sigma}_{\mathrm{A}, \mathrm{c}}$. Further, $E_{x}^{\mathrm{M}}$ will have a finite amplitude at the position of $\bar{\Sigma}_{\mathrm{A}}=\bar{\Sigma}_{\mathrm{A}, \mathrm{c}}$ and its enhancement in the vicinity of the position will be sharp, because it has no singularity everywhere and $\left(j_{z}^{\mathrm{M}}\right)_{\text {ionosphere }}$ changes rapidly across the position. 

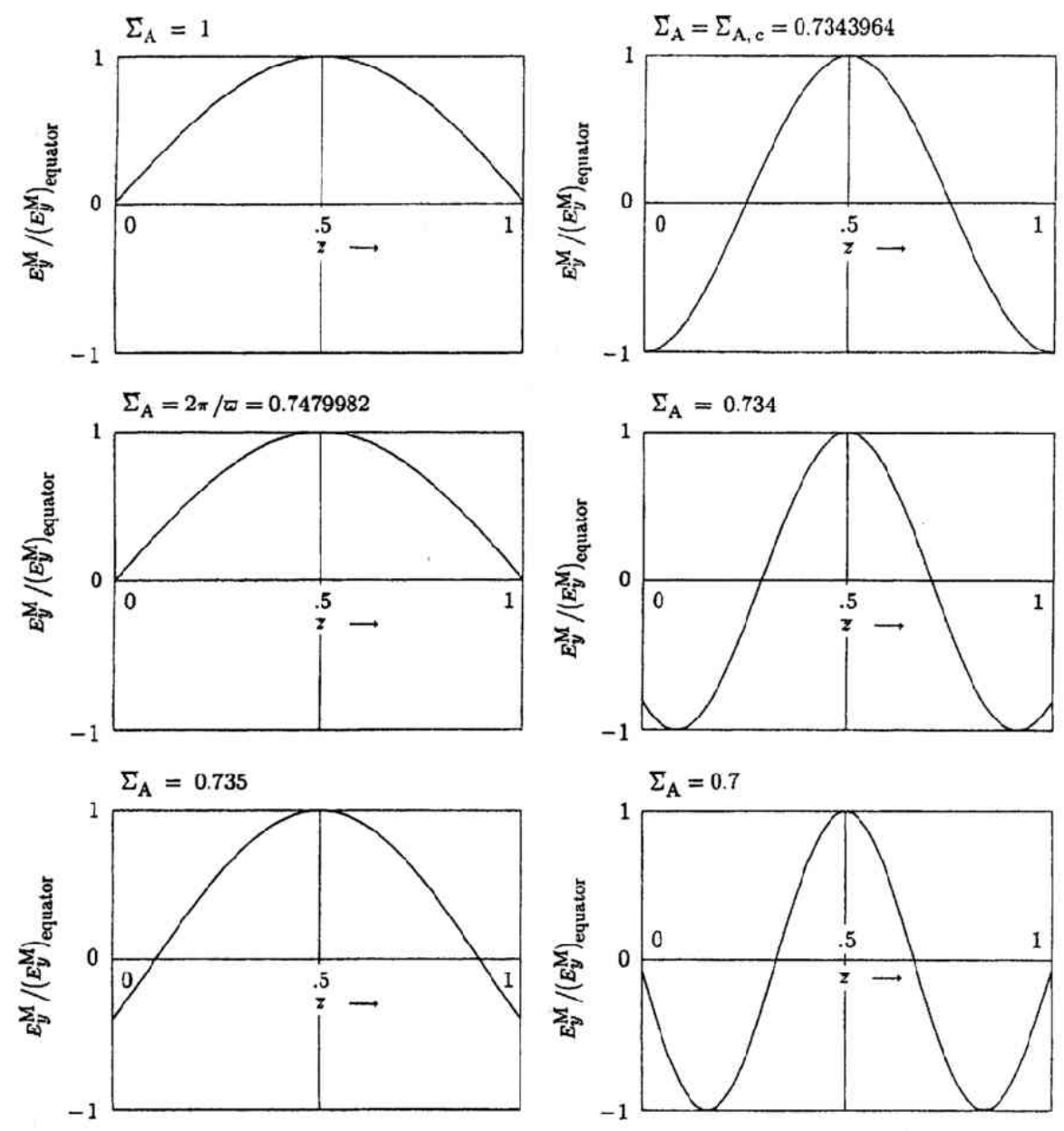

Fig. 5. Parallel structure of the fast mode perturbation $E_{y}^{\mathrm{M}}$ at the field line positions of $\bar{\Sigma}_{\mathrm{A}}=1,2 \pi / \bar{\omega}, 0.735$, $\bar{\Sigma}_{\mathrm{A}, \mathrm{c}}, 0.734$ and 0.7 .

In the case of no energy dissipation ( $\Sigma_{\mathrm{P}}=0$ and $p=i \omega$ ), it is possible for all $G_{2 n}$ 's to be selected as real-valued functions, as seen from Eqs. (28) and (36). As a result of such a selection, the fast mode perturbation $E_{y}^{\mathrm{M}}$ and the Alfvén mode perturbation $E_{x}^{\mathrm{M}}$ have real and purely imaginary values, respectively, everywhere (cf. Eq. (33) with $C_{1}=0$ ). Thus, it is seen that $E_{x}^{\mathrm{M}}$ has no phase shift across its enhancement position in the case of no energy dissipation because it has a finite amplitude at the position.

\section{Discussion}

According to the classical field line resonance theory, since the resonant frequency is determined only from $V_{\mathrm{A}}$ and $\ell_{\|}$, it follows that the resonance with the eigenfrequency $\bar{\omega}$ occurs at the field line position of $\bar{\Sigma}_{\mathrm{A}}=2 \pi / \bar{\omega}$ where the Alfvén mode perturbation $E_{x}^{\mathrm{M}}$ has loops at the ionospheric boundaries owing to $\Sigma_{\mathrm{P}}=0$ and its parallel wavelength is equal to $\ell_{\|}$. Then, the prevailing global cavity mode theory may predict that $E_{x}^{\mathrm{M}}$ is enhanced at the position of $\bar{\Sigma}_{\mathrm{A}}=2 \pi / \bar{\omega}$. In the last section, however, it has been suggested that the enhancement of $E_{x}^{\mathrm{M}}$ 
occurs at the position of $\bar{\Sigma}_{\mathrm{A}}=\bar{\Sigma}_{\mathrm{A}, \mathrm{c}}(<2 \pi / \bar{\omega})$. Thus, it seems that the derived self-consistent ionospheric boundary conditions bring about not only the coupling between the axisymmetric fast and Alfvén waves via the ionospheric Hall current but also the deviation of the location of enhancement of $E_{x}^{\mathrm{M}}$ from that predicted by the classical theory. In the following we will discuss such a deviation.

Yoshikawa et al. (1995) have emphasized that the ionospheric induction current, which appears as the terms associated with $\left(\nabla \times \mathbf{E}_{\perp}^{\mathrm{I}}\right)_{z}$ in the lefthand sides of Eqs. (14) and (23), plays an essential role in the mode conversion between the fast and Alfvén waves in the ionosphere. The ionospheric electric field $\mathbf{E}_{\perp}^{\mathrm{I}}$ can be always separated into the static (or divergent) and inductive (or rotational) parts:

$$
\mathbf{E}_{\perp}^{\mathrm{I}}=-\nabla_{\perp} \varphi-\frac{\partial \mathbf{A}_{\perp}}{\partial t}
$$

where $\varphi$ is a scalar potential and $\mathbf{A}_{\perp}$ a vector one which satisfies $\nabla \cdot \mathbf{A}_{\perp}=0$ (e.g., Tamao, 1986). Then, the ionospheric current is expressed in terms of the divergent current, which closes the field-aligned current (FAC) in the ionosphere, and the eddy (or rotational) current:

$$
\mathbf{J}_{\perp}^{\mathrm{I}}=-\left(\Sigma_{\mathrm{P}} \nabla_{\perp} \varphi+\Sigma_{\mathrm{H}} \frac{\partial \mathbf{A}_{\perp}}{\partial t} \times \mathbf{e}_{z}\right)-\left(\Sigma_{\mathbf{H}} \nabla_{\perp} \varphi \times \mathbf{e}_{z}+\Sigma_{\mathbf{P}} \frac{\partial \mathbf{A}_{\perp}}{\partial t}\right)
$$

which is derived from Eqs. (6) and (48). When both $\Sigma_{\mathrm{P}}$ and $\Sigma_{\mathbf{H}}$ are horizontally uniform, the first and second terms in the righthand side of Eq. (49) are the divergent and eddy parts of the ionospheric current, respectively. Here, as in most of previous works on the field line resonance, let us consider the situation that the ionospheric induction current associated with the vector potential is neglected, which corresponds to using only Eq. (14) together with the assumption of the irrotational ionospheric electric field (Eq. (2)) instead of the derived self-consistent conditions. It should be noted that using only Eq. (14) together with Eq. (2) is not equivalent to only imposing the irrotationality on the self-consistent conditions and the assumption of irrotationality harms the self-consistency of those conditions. Then, the eddy current is only the Hall current driven by the static electric field and is independent of the closed circuit composed of the FAC and the Pedersen current, that is, the temporal variation of the magnetic flux produced by the eddy current has no effect on the closed circuit. In such a situation, therefore, the resonant frequency of field line is determined only from $V_{\mathrm{A}}$ and $\ell_{\|}$. On the other hand, if the induction current is taken into account, the eddy current is no longer independent of the closed circuit which comprises the FAC and its closing current in the ionosphere, because the Hall part of the induction current $\left(-\Sigma_{\mathbf{H}} \partial \mathbf{A}_{\perp} / \partial t \times \mathbf{e}_{z}\right)$ flows as a divergent current and so can contribute to the closing current to the FAC. Tamao $(1975,1984)$ discussed the time response of the magnetosphere-ionosphere current system in terms of an equivalent LCR circuit model. Taking into account the divergent Hall current (Hall part of the induction current) corresponds to adding an extra LR circuit to the LCR one, where they are interconnected via a mutual inductance. Thus, the divergent Hall current will control the time constant of the current system effectively.

Further, Yoshikawa et al. $(1994,1995)$ have found the control of eigenfrequency by $\Sigma_{\mathrm{H}}$ in the localized toroidal oscillation:

1. In the case of insulator-like ionosphere, the eigenfrequency of the fundamental decreases monotonously as $\Sigma_{\mathbf{H}}$ increases.

2. The eigenfrequency of any $n$th harmonic except the fundamental for the insulator-like ionosphere becomes smaller as $\Sigma_{\mathrm{H}}$ grows large, and eventually converges to the value of eigenfrequency of the $(n-1)$ th harmonic for the perfectly conducting ionosphere.

3. In the case of conductor-like ionosphere, for all harmonics including the fundamental, the eigenfrequency first increases with $\Sigma_{\mathrm{H}}$, then turns to decreasing when $\Sigma_{\mathrm{H}}$ exceeds a critical value, and eventually converges to the value for the perfectly conducting ionosphere. 
Here, the insulator-like and conductor-like ionospheres indicate $\Sigma_{\mathrm{P}}\left\langle\Sigma_{\mathrm{A}}\right.$ and $\left.\Sigma_{\mathrm{P}}\right\rangle \Sigma_{\mathrm{A}}$, respectively, for a given magnetic shell. Then, also in case of the second harmonic localized toroidal oscillation for $\Sigma_{P}=0$ which is symmetric about the magnetic equator, it is expected that the eigenfrequency becomes smaller as $\Sigma_{\mathrm{H}}$ grows large, and eventually converges to the value of eigenfrequency of the fundamental one for the perfectly conducting ionosphere. The resonant frequency of field line at the position of $\bar{\Sigma}_{\mathrm{A}}=\bar{\Sigma}_{\mathrm{A}, \mathrm{c}}$ predicted by the classical theory is greater than the given fundamental eigenfrequency $\bar{\omega}$ of magnetospheric cavity oscillation. It is, however, inferred from the control of eigenfrequency found by Yoshikawa et al. $(1994,1995)$ that in the case of insulator-like ionosphere, increasing $\Sigma_{\mathrm{H}}$ is equivalent to lengthening the resonating field line. Then, the actual resonant frequency at the position of $\bar{\Sigma}_{\mathrm{A}}=\bar{\Sigma}_{\mathrm{A}, \mathrm{c}}$ becomes less than the value predicted by the classical theory owing to the divergent Hall current and so it follows that $E_{x}^{\mathrm{M}}$ is enhanced at the position.

The dependence of $\bar{\Sigma}_{\Lambda, c}$ on $\bar{\Sigma}_{\mathbf{H}}$ is determined from

$$
f\left(\bar{\omega} \bar{\Sigma}_{\mathrm{A}, \mathrm{c}}\right)=-\bar{d} \bar{\omega}^{2} \bar{\Sigma}_{\mathbf{H}^{2}}
$$

which is equivalent to Eq. (40), where $f$ is given by Eq. (45). Then, it is seen from Fig. 3 that $\bar{\Sigma}_{\mathrm{A}, \mathrm{c}}$ approaches $2 \pi / \bar{\omega}$ as $\bar{\Sigma}_{\mathrm{H}}$ vanishes, decreases as $\bar{\Sigma}_{\mathrm{H}}$ increases, and converges to $\pi / \bar{\omega}$ as $\bar{\Sigma}_{\mathrm{H}}$ diverges. Here, the parallel wavelength of $E_{x}^{\mathrm{M}}$ is $\ell_{\|}$at the position of $\bar{\Sigma}_{\mathrm{A}}=2 \pi / \bar{\omega}$, indicating that $E_{x}^{\mathrm{M}}$ corresponds to the second harmonic toroidal oscillation in the insulator-like ionosphere, and is $2 \ell_{\|}$at the position of $\bar{\Sigma}_{\mathrm{A}}=\pi / \bar{\omega}$, indicating that $E_{x}^{\mathrm{M}}$ corresponds to the fundamental one in the conductor-like ionosphere. This is well consistent with the second result about the control of eigenfrequency found by Yoshikawa et al. $(1994,1995)$.

It seems that if $\ell_{\perp}<d$, where $\ell_{\perp}$ is a scale of the horizontal spatial variation in the localized toroidal oscillation, the effect of the divergent Hall current can be neglected (Miura et al., 1982). However, the magnetic disturbance with such a horizontal scale is shielded from the ground by the atmosphere (Hughes and Southwood, 1976). Then, so far as the localized toroidal oscillation with an observable ground magnetic effect is concerned, it may be thought that $\ell_{\perp}$ is sufficiently large compared with $d$ and so the divergent Hall current can play a significant role in the oscillation.

\section{Summary}

We have derived the self-consistent ionospheric boundary conditions which describe the interaction between HM waves and the anisotropically conducting ionosphere. These conditions consist of the continuity of the horizontal perturbation electric field across the ionosphere (Eq. (4)), the divergence of a vector formula (Eq. (5)) relating a jump of the horizontal perturbation magnetic field across the ionosphere to the sheet current flowing therein (Eq. (14)) and the normal component of the rotation of the vector formula to the ionosphere (Eq. (15) or Eq. (23)). Equation (23) is preferable in the eigenmode analysis of coupled HM waves in the magnetosphere-ionosphere system to Eq. (15).

In order to emphasize a significant role of the derived self-consistent conditions, assuming that there exists no parallel inhomogeneity but a radial one of the Alfvén velocity $V_{\mathrm{A}}$ and the ionospheric conductivities in both hemispheres are uniform and symmetric, we have examined the coupling between the axisymmetric fast and Alfvén waves via the ionospheric Hall current in case of no energy dissipation (zero Pedersen conductivity). Then, it is suggested that for a given fundamental eigenfrequency, the Alfvén mode perturbation is sharply enhanced with a finite amplitude at a field line position which is deviated from the position predicted by the classical field line resonance theory that the resonant frequency is determined only from the distribution of $V_{\mathrm{A}}$ along the field line and its length $\ell_{\|}$between the northern and southern ionospheres. Such a deviation is due to the divergent Hall current and occurs toward the direction in which 
$V_{\mathrm{A}}$ increases. Further, the Alfvén mode perturbation has no phase shift across the enhancement position. It is also suggested that the parallel wavelength of the fast mode perturbation is slightly greater than $2 \ell_{\|}$in the region with smaller $V_{\mathrm{A}}$ and $2 \ell_{\|} / 3$ in the region with larger $V_{\mathrm{A}}$, respectively, and it changes rapidly from $\sim 2 \ell_{\|}$to $\sim 2 \ell_{\|} / 3$ through $\ell_{\|}$as $V_{\mathrm{A}}$ grows large, where the wavelength $\ell_{\|}$appears just at the enhancement position.

In order to confirm the above suggestions it is needed to solve Eq. (28) under the constraint of Eq. (36), and this will be done as a future work.

The authors would like to express their thanks to Dr. S. Fujita for his helpful discussions. They are also greatly indebted to an anonymous referee for his illuminative suggestions and criticism. This work was partly supported by the Grant-in-Aid for Scientific Research (C), No. 05640488, from The Ministry of Education, Science and Culture.

\section{REFERENCES}

Allan, W. and F. B. Knox, A dipole field model for axisymmetric Alfvén waves with finite ionosphere conductivities, Planet. Space Sci., 27, 79 85, 1979.

Allan, W., S. P. White, and E. M. Poulter, Magnetospheric coupling of hydromagnetic waves - initial results, Geophys. Res. Lett., 12, 287-290, 1985.

Allan, W., S. P. White, and E. M. Poulter, Impulse-excited hydromagnetic cavity and field-line resonances in the magnetosphere, Planet. Space Sci., 34, 371-385, 1986.

Fujita, S., Coupled magnetohydrodynamic oscillations in the magnetosphere-ionosphere system-Hall current effect, Proc. NIPR Symp. Upper Atmos. Phys., 6, 76-89, 1993.

Fujita, S. and V. L. Patel, Eigenmode analysis of coupled magnetohydrodynamic oscillations in the magnetosphere, J. Geophys. Res., 97, 13,777-13,788, 1992.

Glassmeier, K. H., Reflection of MHD-waves in the Pc4-5 period range at ionospheres with non-uniform conductivity distributions, Geophys. Res. Lett., 10, 678-681, 1983.

Glassmeier, K. H., On the influence of ionospheres with non-uniform conductivity distribution on hydromagnetic waves, J. Geophys., 54, 125-137, 1984.

Hughes, W. J. and D. J. Southwood, The screening of micropulsation signals by the atmosphere and ionosphere, J. Geophys. Res., 81, 3234-3240, 1976.

Kivelson, M. G. and D. J. Southwood, Resonant ULF waves: A new interpretation, Geophys. Res. Lett., 12, 49-52, 1985.

Kivelson, M. G. and D. J. Southwood, Coupling of global magnetospheric MHD eigenmodes to field line resonances, J. Geophys. Res., 91, 4345 4351, 1986.

Kivelson, M. G. and D. J. Southwood, Hydromagnetic waves and the ionosphere, Geophys. Res. Lett., 15, 1271$1274,1988$.

Kivelson, M. G., J. Etcheto, and J. G. Trotignon, Global compressional oscillations of the terrestrial magnetosphere: The evidence and a model, J. Geophys. Res., 89, 9851-9856, 1984.

Lee, D.-H. and R. L. Lysak, Magnetospheric ULF wave coupling in the dipole model: The impulsive excitation, J. Geophys. Res., 94, 17,097-17,103, 1989.

Lee, D.-H. and R. L. Lysak, Effects of azimuthal asymmetry on ULF waves in the dipole magnetosphere, Geophys. Res. Lett., 17, 53-56, 1990.

Miura, A., S. Ohtsuka, and T. Tamao, Coupling instability of the shear Alfvén wave in the magnetosphere with ionospheric ion drift wave, 2. Numerical analysis, J. Geophys. Res., 87, 843-851, 1982.

Newton, R. S., D. J. Southwood, and W. J. Hughes, Damping of geomagnetic pulsations by the ionosphere, Planet. Space Sci., 26, 201-209, 1978.

Orr, D. and J. A. D. Matthew, The variation of geomagnetic micropulsation periods with latitude and the plasmapause, Planet. Space Sci., 19, 897-905, 1971.

Samson, J. C., and G. Rostoker, Latitude-dependent characteristics of high-latitude Pc 4 and Pc 5 micropulsations, J. Geophys. Res., 77, 6133-6144, 1972.

Tamao, T., Unsteady interactions of solar wind disturbances with the magnetosphere, J. Geophys. Res., 80, 4230-4236, 1975.

Tamao, T., Magnetosphere-ionosphere interaction through magnetohydrodynamic waves, Proc. Symp. on Achievements of the IMS, Spec. Publ. 217, pp. 427-435, Eur. Space Agency, Neuilly, France, 1984.

Tamao, T., Direct contribution of oblique field-aligned currents to ground magnetic fields, J. Geophys. Res., 91, 183-189, 1986.

Yoshikawa, A., M. Itonaga, and T.-I. Kitamura, On the coupled effect between the field-aligned and ionospheric 
induction currents, Proceedings of 8th International Symposium on Solar Terrestrial Physics, Part I, p. 155, SCOSTEP, Sendai, Japan, 1994

Yoshikawa, A., M. Itonaga, and T.-I. Kitamura, Effect of the ionospheric induction current on magnetohydrodynamic waves in the magnetosphere, Proc. NIPR Symp. Upper Atmos. Phys., 8, 49-59, 1995. 